\title{
Comparação entre Variantes do Método de Pontos Interiores para Multifluxo
}

V. PODESTÁ-GOMES, C. PERIN, Departamento de Matemática Aplicada, Instituto de Matemática, Estatística e Computação Científica, Universidade Estadual de Campinas, UNICAMP, Cx.P. 6065, 13083-970 Campinas, SP, Brasil.

Resumo. Apresentamos uma comparação entre duas versões do método primaldual de pontos interiores especializado para multifluxo: uma versão que chamamos de Comum e outra versão conhecida por Preditor-Corretor. Comparamos estas duas versões quanto ao tempo de cpu, número de iterações e número de iterações do gradiente conjugado precondicionado, na presença de diferentes pontos iniciais e de inicializações distintas no gradiente conjugado.

\section{Formulação do Problema}

O Problema de Multifluxo consiste em encontrar uma solução de mínimo custo total de transporte de $p$ produtos através de uma rede com $m$ nós e $n$ arcos, onde os arcos possuem uma capacidade individual para cada produto e também uma capacidade mútua, compartilhada por todos os produtos. Além disso, existem as restrições usuais de conservação de fluxo para cada produto em cada nó da rede. A formulação mais conhecida para este problema (formulação nó-arco) é:

$$
\begin{array}{ll}
\min & \sum_{k=1}^{p}\left(c^{k}\right)^{\prime} x^{k} \\
\text { s.a. } & \left\{\begin{array}{l}
A x^{k}=b^{k}, k=1, \cdots, p \\
\sum_{k=1}^{p} x^{k} \leq d \\
0 \leq x^{k} \leq u^{k}, k=1, \cdots, p .
\end{array}\right.
\end{array}
$$

Os vetores $x^{k} \in \mathbb{R}^{n}, c^{k} \in \mathbb{R}^{n}, u^{k} \in \mathbb{R}^{n}$ são, respectivamente, os vetores das variáveis de fluxo, de custos unitários de transporte e de capacidade individual para cada produto $k ; b^{k} \in \mathbb{R}^{m}$ é o vetor de ofertas/demandas do produto $k$, em cada nó da rede. $A \in \mathbb{R}^{m \times n}$ é a matriz de incidência: cada uma de suas colunas corresponde a um arco da rede. É bastante conhecido que, se a rede é conectada, então é necessário remover uma linha qualquer para que a matriz $A$ passe a ter posto completo. O primeiro grupo de restrições formam as restrições de oferta/demanda para cada produto. O vetor $d \in \mathbb{R}^{n}$ é o vetor de capacidade mútua dos arcos, e o 
segundo grupo de restrições são as restrições de acoplamento. As últimas restrições são as de canalização sobre as variáveis de fluxo.

Este é um problema de programação linear que, em sua forma padrão possui $\widehat{m}=p(m-1)+n$ restrições e $\widehat{n}=(p+1) n$ variáveis e é considerado um problema de grande porte. No entanto, sua matriz de coeficientes possui uma estrutura bem particular: ela é bloco angular com uma faixa horizontal; cada bloco é formado pela matriz de incidência da rede e a faixa é composta por matrizes identidade. Vários métodos já foram propostos para esta estrutura. Mais recentemente, os métodos de pontos interiores têm se mostrado bastante competitivos e, dentre eles, a versão primal-dual tem mostrado um bom desempenho ([1], [2], [4]), bem com sua versão preditor-corretor ([3], [5]). Neste trabalho, comparamos as duas versões do método primal-dual de pontos interiores especializado para multifluxo: a versão que chamamos de Comum e a versão Preditor-Corretor.

\section{O Método Primal-Dual de Pontos Interiores}

Considere o par primal/dual de problemas lineares, resultante do problema original:

$\begin{array}{ll}\min \quad & \hat{c}^{\prime} \hat{x} \\ \text { s.a. } & \left\{\begin{array}{l}\hat{A} \hat{x}=\hat{b} \\ \hat{x}+\hat{s}=\hat{u} \\ \hat{x}, \hat{s} \geq 0\end{array}\right.\end{array}$

$\max \hat{b}^{\prime} \hat{y}-\hat{u}^{\prime} \hat{w}$

s.a. $\quad\left\{\begin{array}{l}\hat{A}^{\prime} \hat{y}-\hat{w}+\hat{z}=\hat{c} \\ \hat{w}, \hat{z} \geq 0\end{array}\right.$

onde

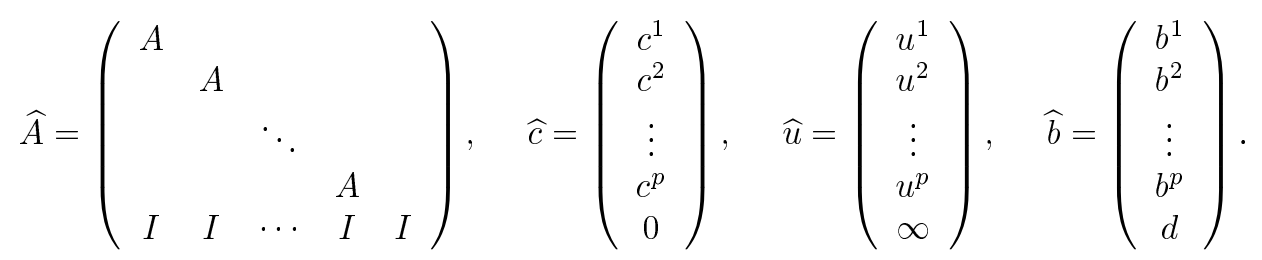

Nesta notação, $\widehat{A} \in R^{\widehat{m} \times \widehat{n}} ; \widehat{c}, \widehat{x}, \widehat{s}, \widehat{u}, \widehat{z}$ e $\widehat{w} \in \mathbb{R}^{\widehat{n}}, \widehat{b}$ e $\widehat{y} \in \mathbb{R}^{\widehat{m}} ; \widehat{y}$ e $\widehat{w}$ são as variáveis duais correspondentes às restrições de conservação de fluxo e de canalização, respectivamente, e $\widehat{z}$ á folga dual. Todos estes vetores são particionados por produtos (ou blocos): uma componente para cada produto $k, k=1, \cdots, p$ e mais uma componente correspondente às restrições de acoplamento.

Resumidamente, o método primal-dual de pontos interiores especializado para multifluxo consiste em, dado um ponto inicial interior $(\widehat{x}, \widehat{s})$ para o problema primal $(\mathrm{P})$, não necessariamente factível, um ponto inicial interior $(\widehat{y}, \widehat{w}, \widehat{z})$ para o problema dual (D), não necessariamente factível e um certo parâmetro dito de centragem, encontrar uma direção de deslocamento para obtermos o próximo ponto. A direção tomada é a de Newton e, de fato, esta é a etapa do método que demanda o maior esforço computacional, onde é necessário resolver um sistema linear com $\widehat{m}$ equações. Obtida esta direção e usando alguma regra para obter o novo parâmetro 
de centragem, repetimos o processo a partir do novo ponto, até que um critério de parada seja satisfeito. No decorrer das iterações, a factibilidade para os problemas primal e dual vai sendo alcançada, bem como a otimalidade.

A diferença entre as duas versões apresentadas está na obtenção da direção de deslocamento. Na versão Comum resolvemos um sistema linear; na versão PreditorCorretor são resolvidos dois sistemas lineares a cada iteração do método primal-dual.

Nos dois casos, estamos utilizando o método do gradiente conjugado precondicionado para resolver os sistemas lineares, onde a estrutura da matriz $\widehat{A}$ é aproveitada. Nesta resolução, usamos uma combinação de dois precondicionadores: o Diagonal, nas primeiras iterações do método primal-dual e o Floresta Geradora Máxima, nas iterações posteriores (ver [6]).

A direção de deslocamento é particionada de acordo com as variáveis primais e duais: $\Delta \widehat{x}, \Delta \widehat{s}, \Delta \widehat{y}, \Delta \widehat{w}, \Delta \widehat{z}$. Desta maneira, o método primal-dual pode ser resumido no seguinte algoritmo:

\section{Algoritmo primal-dual}

- Fornecer um ponto inicial interior $(\widehat{x}, \widehat{s})$ interior para o primal e $(\widehat{y}, \widehat{w}, \widehat{z})$ para o dual.

- Repetir :

obter o parâmetro de centragem $\mu$;

obter a direção de deslocamento $\Delta \widehat{y}$, resolvendo um sistema linear; calcular $\Delta \widehat{x}, \Delta \widehat{s}, \Delta \widehat{w}, \Delta \widehat{z}$ a partir de $\Delta \widehat{y}$;

teste da razão: calcular $\lambda_{P}$ e $\lambda_{D}$;

$(\widehat{x}, \widehat{s}) \leftarrow(\widehat{x}, \widehat{s})+\lambda_{P}(\Delta \widehat{x}, \Delta \widehat{s}) ;$

$(\widehat{y}, \widehat{w}, \widehat{z}) \leftarrow(\widehat{y}, \widehat{w}, \widehat{z})+\lambda_{D}(\Delta \widehat{y}, \Delta \widehat{w}, \Delta \widehat{z}) ;$

até que um critério de parada seja satisfeito.

O passo da obtenção da direção de deslocamento é o que diferencia as versões primal-dual Comum e Preditor-Corretor, além de ser o passo computacionalmente mais caro do algoritmo. Este passo será detalhado a seguir.

\section{Primal-Dual Comum}

Na versão primal-dual Comum, o sistema linear que encontra a direção de deslocamento é:

$$
\underbrace{\left[\begin{array}{rrrrr}
\widehat{A} & 0 & 0 & 0 & 0 \\
I & I & 0 & 0 & 0 \\
0 & 0 & \widehat{A}^{\prime} & -I & I \\
\widehat{Z} & 0 & 0 & 0 & \widehat{X} \\
0 & \widehat{W} & 0 & \widehat{S} & 0
\end{array}\right]}_{\widehat{Q}}\left[\begin{array}{c}
\Delta \widehat{x} \\
\Delta \widehat{s} \\
\Delta \widehat{y} \\
\Delta \widehat{w} \\
\Delta \widehat{z}
\end{array}\right]=\left[\begin{array}{c}
\widehat{b}-\hat{A} \widehat{x} \\
\widehat{u}-\widehat{x}-\widehat{s} \\
\widehat{c}-\widehat{A^{\prime}} \widehat{y}+\widehat{w}-\widehat{z} \\
\mu \mathbf{1}-\widehat{X} \widehat{Z} \mathbf{1} \\
\mu \mathbf{1}-\widehat{S W} \mathbf{1}
\end{array}\right],
$$


onde $\widehat{X}=\operatorname{diagonal}\left(X^{1}, \cdots, X^{p}, X^{p+1}\right), \operatorname{com} X^{k}=\operatorname{diagonal}\left(x_{j}^{k}\right), \forall k, j=1, \cdots, n$; $\widehat{S}, \widehat{W}, \widehat{Z}$ têm definições similares à matriz $\widehat{X}$, isto é, cada uma delas é uma matriz diagonal por blocos com $\widehat{n}$ elementos positivos (matrizes formadas pelas respectivas soluções correntes) e $\mathbf{1}$ é o vetor coluna com $\widehat{n}$ componentes iguais a 1 .

Com algumas manipulações nas equações deste sistema, ele fica reduzido a um sistema linear de dimensão bem menor, $\widehat{m}$, que é

$$
\begin{aligned}
\left(\widehat{A} \widehat{\Theta} \widehat{A}^{\prime}\right) \Delta \widehat{y}= & \widehat{A} \widehat{\Theta}\left\{\left[\mu\left((\widehat{S})^{-1}-(\widehat{X})^{-1}\right)-\widehat{W}+\widehat{Z}\right] \mathbf{1}+\left(\widehat{c}-\widehat{A}^{\prime} \widehat{y}+\widehat{w}-\widehat{z}\right)-\right. \\
& \left.(\widehat{S})^{-1} \widehat{W}(\widehat{u}-\widehat{x}-\widehat{s})\right\}+(\widehat{b}-\widehat{A} \widehat{x})
\end{aligned}
$$

ou, de uma maneira mais conveniente,

$$
B \Delta \widehat{y}=r,
$$

onde a matriz $\widehat{\Theta}=\left[(\widehat{X})^{-1} \widehat{Z}+(\widehat{S})^{-1} \widehat{W}\right]^{-1}$ é uma matriz diagonal, com elementos positivos, denominada matriz de scaling do método primal-dual.

A matriz $B$ do sistema linear (2.2) é simétrica e definida positiva, sendo então adequado o uso do gradiente conjugado precondicionado na resolução deste sistema.

As outras componentes da direção $(\Delta \widehat{x}, \Delta \widehat{s}, \Delta \widehat{w}, \Delta \widehat{z})$ podem ser obtidas a partir de $\Delta \widehat{y}(\operatorname{ver}[6])$.

\section{Preditor-Corretor}

Esta variante do método primal-dual foi apresentada por Mehrotra [5] e Lustig, Marsten, Shanno [3]. A diferença fundamental entre o método primal-dual Comum e esta variante está na maneira de calcular a direção de deslocamento: no PreditorCorretor, a direção é obtida resolvendo dois sistemas lineares a cada iteração, onde a matriz dos coeficientes é a mesma, mas os lados direitos são distintos.

Primeiro resolvemos

$$
\widehat{Q} \bar{\Delta}=\bar{q}
$$

onde $\widehat{Q}$ é a mesma matriz dos coeficientes do sistema linear (2.1), as novas variáveis são $\bar{\Delta}=(\bar{\Delta} \widehat{x}, \bar{\Delta} \widehat{s}, \bar{\Delta} \widehat{y}, \bar{\Delta} \widehat{w}, \bar{\Delta} \widehat{z})$, chamadas de direções afins e o lado direito é $\bar{q}=\left(\widehat{b}-\widehat{A} \widehat{x}, \quad \widehat{u}-\widehat{x}-\widehat{s}, \widehat{c}-\widehat{A}^{\prime} \widehat{y}+\widehat{w}-\widehat{z}, \quad \widehat{X} \widehat{Z} \mathbf{1}, \widehat{S W} \mathbf{1}\right)$.

O segundo sistema linear a ser resolvido é

$$
\widehat{Q} \Delta=\widehat{q},
$$

com as variáveis $\Delta=(\Delta \widehat{x}, \Delta \widehat{s}, \Delta \widehat{y}, \Delta \widehat{w}, \Delta \widehat{z})$ e lado direito $\widehat{q}=\left(\widehat{b}-\widehat{A} \widehat{x}, \widehat{u}-\widehat{x}-\widehat{s}, \widehat{c}-\widehat{A^{\prime}} \widehat{y}+\widehat{w}-\widehat{z}, \mu \mathbf{1}-\widehat{X} \widehat{Z} \mathbf{1}-\bar{\Delta} \widehat{x} \bar{\Delta} \widehat{z} \mathbf{1}, \mu \mathbf{1}-\widehat{S} \widehat{W} \mathbf{1}-\bar{\Delta} \widehat{s} \bar{\Delta} \widehat{w} \mathbf{1}\right)$.

Note que temos a mesma matriz $\widehat{Q}$ nos dois sistemas lineares. A diferença entre eles está apenas no lado direito: nas duas últimas componentes do segundo sistema temos a presença dos termos não lineares $\bar{\Delta} \widehat{x} \bar{\Delta} \widehat{z}$ e $\bar{\Delta} \widehat{s} \bar{\Delta} \widehat{w}$. 
Esta variante reduz o número de iterações do algoritmo anterior, mas exige que resolvamos agora dois sistemas lineares em cada iteração do método primal-dual. No entanto, como a matriz destes sistemas é a mesma matriz $\widehat{Q}$, os cálculos utilizados para construí-la (ou parte dela) são efetuados uma única vez.

Transformações similares às que foram feitas para transformar o sistema linear (2.1) no sistema reduzido (2.2) também podem ser efetuadas nesta variante, de modo que os dois sistemas lineares resolvidos em cada iteração do primal-dual possuem a mesma dimensão do sistema (2.2), isto é, $\widehat{m}$ equações e incógnitas, sendo também um sistema simétrico e definido positivo, onde podemos utilizar o gradiente conjugado precondicionado.

\section{Pontos Iniciais}

\section{Ponto inicial I:}

Este ponto inicial está descrito em [6]. Começamos com $\left(\widehat{x}^{0}, \widehat{s}^{0}, \widehat{y}^{0}, \widehat{w}^{0}, \widehat{z}^{0}\right)$ que satizfaz $\widehat{A}^{\prime} \widehat{y}^{0}-\widehat{w}^{0}+\widehat{z}^{0}=\widehat{c}, \widehat{w}^{0}, \widehat{z}^{0}>0$ (dual factível); $\widehat{x}^{0}, \widehat{s}^{0}>0, \widehat{x}^{0}+\widehat{s}^{0}=\widehat{u}$.

Solução dual: consideramos $\widehat{y}^{0}$ um vetor onde $\left(y^{k}\right)^{0}$ seja qualquer, $k=1, \cdots, p$, mas $\left(y^{p+1}\right)^{0}<0$. Para cada bloco $k$, e para cada arco $a_{t}=(i, j), t=1, \cdots, n$ da rede, calculamos:

$$
\tilde{c}_{i j}^{k}=c_{i j}^{k}-\left(y_{i}^{k}\right)^{0}+\left(y_{j}^{k}\right)^{0}-\left(y_{t}^{p+1}\right)^{0} .
$$

Seja $M=\max _{k,(i j)}\left\{\left|\tilde{c}_{i j}^{k}\right|\right\}+\max _{t}\left\{\left(\mid y_{t}^{p+1}\right)^{0} \mid\right\}, \forall k, \forall(i, j)$.

Para garantirmos $\widehat{w}^{0}>0$ e $\widehat{z}^{0}>0$, tomamos, para $\forall k$ e $\forall(i, j)$ :

se $\tilde{c}_{i j}^{k}>0$, então $\left(w_{t}^{k}\right)^{0}=M$ e $\left(z_{t}^{k}\right)^{0}=\tilde{c}_{i j}^{k}+M$,

se $\tilde{c}_{i j}^{k} \leq 0$, então $\left(z_{t}^{k}\right)^{0}=M$ e $\left(w_{t}^{k}\right)^{0}=-\tilde{c}_{i j}^{k}+M$.

Desta maneira, todas as restrições duais ficam satisfeitas. Em nossa implementação, iniciamos com $\left(y^{k}\right)^{0}=0, k=1, \cdots, p$ e $\left(y^{p+1}\right)^{0}=-(1,1, \cdots, 1)^{\prime}$, de modo que $\tilde{c}_{i j}^{k}=c_{i j}^{k}+1, \quad \forall k, \quad \forall(i, j)$.

Solução primal: para cada arco $a_{t}=(i, j)$ calculamos soma $=\sum_{k=1}^{p} u_{t}^{k}$. Se soma $<2 d_{t}$, então $\left(x_{t}^{k}\right)^{0}=u_{t}^{k} / 2 ;$ senão, $\left(x_{t}^{k}\right)^{0}=\left(u_{t}^{k} d_{t}\right) / 2$ soma, $k=1, \cdots, p$. $\left(x_{t}^{p+1}\right)^{0}=d_{t}-\sum_{k=1}^{p}\left(x_{t}^{k}\right)^{0}, \quad t=1, \cdots, n$ e $\widehat{s}^{0}=\widehat{u}-\widehat{x}^{0}$.

Assim, as únicas equações que poderão não estar satisfeitas são as de conservação de fluxo nos nós dos produtos $\left(A x^{k}=b^{k}\right)$.

\section{Ponto inicial II:}

Este ponto inicial é uma extensão do ponto sugerido por Mehrotra para problemas gerais de Programação Linear, e sua descrição pode ser encontrada em [5]. Não sendo específico para redes, ele deixa de tirar proveito da estrutura de nosso problema. Este ponto requer um trabalho maior para ser construído: é necessário resolver dois sistemas lineares de dimensão $\widehat{m}$ para a obtenção de $\widehat{x}^{0}$ e de $\widehat{y}^{0}$. 


\section{Experimentos Computacionais}

Construímos um gerador de problemas de multifluxo e, através dele, vários testes computacionais foram realizados. Os problemas são gerados a partir da especificação de um tamanho e de uma semente. A rede associada ao problema é conectada e os vetores $u^{k}$ e $c^{k}, \forall k$, são gerados com distribuição uniforme em intervalos cujos limites são fornecidos. Nestes experimentos, consideramos $c_{j}^{k} \in[0,5], u_{j}^{k} \in[1,5], \forall k, \forall j$. Além disso, temos também a opção de deixar as restrições de acoplamento mais "apertadas" ou mais "folgadas" (vetor $d$ ).

A implementação está feita na linguagem $C^{++}$, com dupla precisão. Todos os experimentos foram realizados numa Sun Ultra SPARC 10, com $300 \mathrm{MHz}$ e 128 Mbytes de memória RAM.

Utilizando os pontos iniciais I e II e variando a inicialização do gradiente conjugado precondicionado, medimos o tempo de cpu, o número total de iterações do primal-dual e o número total de iterações do gradiente conjugado.

\begin{tabular}{|c|c|c|c|c|c|}
\hline prob. & $m$ & $n$ & $p$ & $\widehat{m}$ & $\widehat{n}$ \\
\hline 1 & 50 & 101 & 10 & 591 & 1111 \\
2 & 100 & 201 & 10 & 1191 & 2211 \\
3 & 200 & 306 & 10 & 2296 & 3366 \\
4 & 200 & 401 & 10 & 2391 & 4411 \\
5 & 200 & 500 & 10 & 2490 & 5500 \\
6 & 300 & 507 & 10 & 3497 & 5577 \\
7 & 300 & 604 & 10 & 3594 & 6644 \\
8 & 400 & 620 & 10 & 4610 & 6820 \\
9 & 400 & 805 & 10 & 4795 & 8855 \\
10 & 500 & 902 & 10 & 5901 & 9922 \\
11 & 500 & 1009 & 10 & 5999 & 11099 \\
12 & 600 & 1212 & 10 & 7202 & 13332 \\
13 & 800 & 1515 & 10 & 9505 & 16665 \\
14 & 1000 & 2017 & 10 & 12007 & 22187 \\
15 & 1000 & 3006 & 10 & 12996 & 33066 \\
16 & 2000 & 3100 & 10 & 23090 & 34100 \\
\hline 17 & 100 & 201 & 20 & 2181 & 4221 \\
18 & 200 & 408 & 20 & 4388 & 8568 \\
19 & 300 & 516 & 20 & 6496 & 10836 \\
20 & 400 & 814 & 20 & 8794 & 17094 \\
21 & 1000 & 2028 & 20 & 22008 & 42588 \\
22 & 2000 & 4044 & 20 & 44024 & 84924 \\
\hline
\end{tabular}

Tabela 1: Problemas gerados

A Tabela 1 apresenta os problemas gerados para este trabalho. Nos problemas de 1 a 16, aumentamos o tamanho da rede $(m$ e $n)$ mantendo o número de produtos $(p)$ em 10; nos problemas de 17 a 22, mantivemos o número de produtos em 20; $\widehat{m}=p(m-1)+n$ é a dimensão do sistema linear que é resolvido em cada iteração do primal-dual; $\widehat{n}=(p+1) n$ é o número de variáveis do problema de multifluxo. Os resultados a seguir se referem a estes 22 problemas. 
Em todos estes resultados, utilizamos a melhor combinação que encontramos entre a inicialização do gradiente conjugado precondicionado e a utilização das duas opções de ponto inicial, ou seja:

- com o ponto inicial I, o melhor é iniciar o gradiente conjugado precondicionado com a direção nula, nas duas versões do primal-dual;

- com o ponto inicial II, e na versão Comum, como é praticamente indiferente iniciar o gradiente conjugado precondicionado com a direção nula ou com a direção obtida pelo gradiente conjugado na iteração anterior do primal-dual, optamos por utilizar a direção nula, que é mais simples. Na versão Preditor-Corretor, o melhor é iniciar o Corretor com a direção obtida pelo Preditor. Neste caso, ainda é um pouco melhor iniciar o Preditor com a direção nula.

Comparação entre o número total de iterações do primal-dual: neste item, pudemos concluir claramente que o número de iterações na versão Preditor-Corretor é sempre menor do que na versão Comum, independentemente do ponto inicial utilizado. No gráfico dado pela Figura 1, onde usamos o ponto inicial II, este fato pode ser observado em todos os problemas (os resultados são similares para o ponto inicial I). O número máximo de iterações obtido com o Preditor-Corretor ocorreu no problema 22 (32 iterações) enquanto que, na versão Comum, este número ficou em 41 (problema 22).

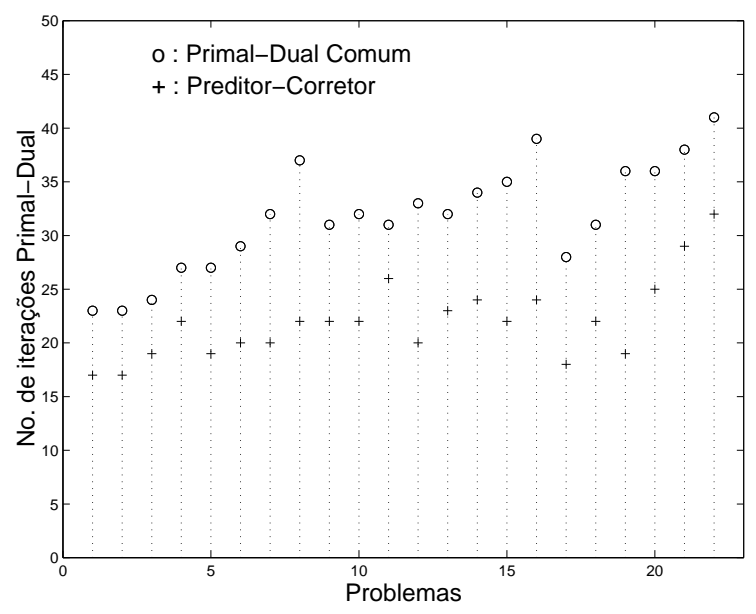

Figura 1: comparação entre no. de iterações Primal-Dual (ponto inicial II)

Comparação entre tempos de CPU: nas colunas 2, 3, 4 e 5 da Tabela 2 temos o tempo de CPU, em segundos, quando rodamos as versões primal-dual Comum e Preditor-Corretor, na presença dos dois pontos iniciais descritos (colunas "p.i. I" e "p.i. II"). Comparando as duas versões do primal-dual, vemos que na versão Comum, 16 problemas apresentaram tempo de CPU menor quando utilizamos o ponto inicial I e, na versão Preditor-Corretor, 14 problemas. Comparando agora 


\begin{tabular}{|c||c|c|c|c||c|c|c|c|}
\hline \multicolumn{1}{|c||}{} & \multicolumn{4}{|c||}{ Tempo de CPU } & \multicolumn{3}{c|}{ Total de it. do Grad.Conj. } \\
\cline { 2 - 8 } pr. & PD Comum & Pred.-Corretor & PD Comum & \multicolumn{2}{c|}{ Pred.-Corretor } \\
\cline { 2 - 8 } & p.i. I & p.i. II & p.i. I & p.i. II & p.i. I & p.i. II & p.i. I & p.i. II \\
\hline \hline 1 & 8 & 10 & 14 & 14 & 1060 & 920 & 1952 & 1564 \\
2 & 31 & 33 & 38 & 34 & 1886 & 1725 & 2592 & 2023 \\
3 & 82 & 86 & 119 & 127 & 2712 & 2568 & 4680 & 4313 \\
4 & 137 & 136 & 164 & 168 & 4230 & 3888 & 5412 & 5038 \\
5 & 94 & 107 & 108 & 117 & 2575 & 2565 & 3150 & 3078 \\
6 & 208 & 241 & 218 & 244 & 4550 & 4930 & 5112 & 5240 \\
7 & 296 & 369 & 286 & 253 & 6102 & 7232 & 6156 & 4920 \\
8 & 408 & 732 & 594 & 703 & 6858 & 12210 & 10258 & 11902 \\
9 & 438 & 549 & 405 & 481 & 6696 & 7719 & 6400 & 6908 \\
10 & 1053 & 902 & 689 & 820 & 13330 & 11136 & 8862 & 10010 \\
11 & 1167 & 1138 & 1118 & 1308 & 14570 & 13578 & 13777 & 16016 \\
12 & 1705 & 1592 & 1547 & 1040 & 17329 & 15642 & 15768 & 10140 \\
13 & 1939 & 2255 & 2187 & 2071 & 14520 & 16448 & 16654 & 15295 \\
14 & 5286 & 5045 & 3284 & 5154 & 30987 & 28968 & 19780 & 29424 \\
15 & 4150 & 4787 & 4665 & 2852 & 20160 & 22855 & 23046 & 13552 \\
16 & 19485 & 19974 & 23588 & 13870 & 60830 & 62556 & 74400 & 43344 \\
\hline 17 & 16 & 27 & 27 & 30 & 1334 & 1680 & 2175 & 1692 \\
18 & 76 & 97 & 110 & 131 & 3094 & 3224 & 4142 & 3960 \\
19 & 142 & 219 & 166 & 192 & 3942 & 5076 & 4275 & 3800 \\
20 & 492 & 497 & 1009 & 528 & 9548 & 8748 & 16640 & 7675 \\
21 & 3040 & 3258 & 2101 & 3022 & 22500 & 31084 & 16011 & 22475 \\
22 & 17473 & 10347 & 21876 & 19297 & 6440 & 37105 & 80820 & 71776 \\
\hline
\end{tabular}

Tabela 2: Tempo de CPU e Total de it. do Gradiente Conjugado

os dois pontos iniciais: utilizando o ponto inicial I, a versão Comum apresentou 15 problemas com tempo de CPU menor. Utilizando o ponto inicial II, a versão Comum apresentou 12 problemas com tempo de CPU menor.

Total de iterações no Gradiente Conjugado: nas colunas 6, 7, 8 e 9 da Tabela 2 temos o número total de iterações no gradiente conjugado precondicionado, com as versões Comum e Preditor-Corretor, utilizando os pontos iniciais I e II. Na versão Preditor-Corretor, o total de iterações do gradiente conjugado é a soma do número de iterações do Preditor com o número de iterações do Corretor. Comparando as duas versões do primal-dual, na versão Comum tivemos 12 problemas com um total de iterações no gradiente conjugado menor quando utilizamos o ponto inicial I e, na versão Preditor-Corretor, tivemos apenas 8 problemas. Comparando os dois pontos iniciais: com o ponto inicial I, a versão Comum apresentou 16 problemas com um total de iterações no gradiente conjugado menor; com o ponto inicial II, as duas versões se comportaram igualmente.

Na Figura 2 temos a razão entre os tempos de CPU (CPU do PreditorCorretor/CPU do Comum) na presença do ponto inicial I (um gráfico muito semelhante é obtido quando utilizamos o ponto inicial II). Aqui, podemos observar mais 
claramente que a versão Comum apresenta um comportamento melhor. A Figura 3 mostra a razão entre o total de iterações do gradiente conjugado (total de iterações do Preditor-Corretor/total de iterações do Comum) na presença do ponto inicial I. Novamente, a versão Comum apresenta um comportamento melhor.

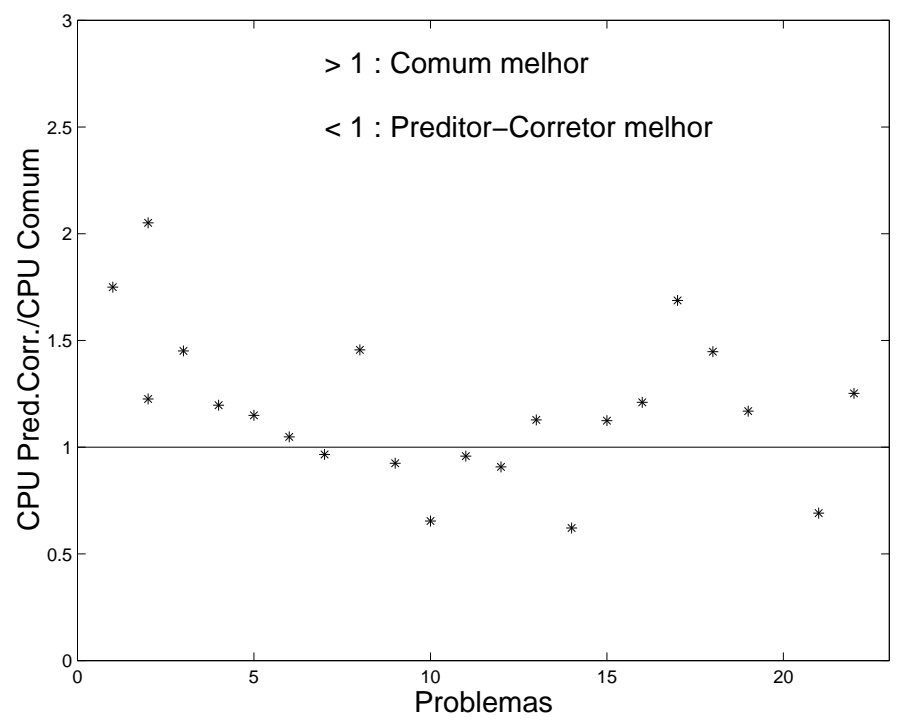

Figura 2: razão entre tempos de CPU (ponto inicial I)

\section{Comentários Finais}

Quanto ao número de iterações primal-dual, a versão Preditor-Corretor apresentou um menor número de iterações do que a versão Comum, como já era esperado. Além disso, tanto na versão Comum quanto na Preditor-Corretor, o ponto inicial I apresentou um melhor comportamento.

Quanto ao tempo de CPU, a combinação que apresentou tempos menores foi a versão Comum e o ponto inicial I. Assim, um número menor de iterações no primal-dual não implica necessariamente numa redução do tempo de CPU.

Quanto ao número total de iterações do gradiente conjugado precondicionado, os experimentos não são conclusivos: parece ser um pouco melhor utilizar o ponto inicial II na versão Preditor-Corretor (como sugerido por Mehrotra) e o ponto inicial I na versão Comum. 


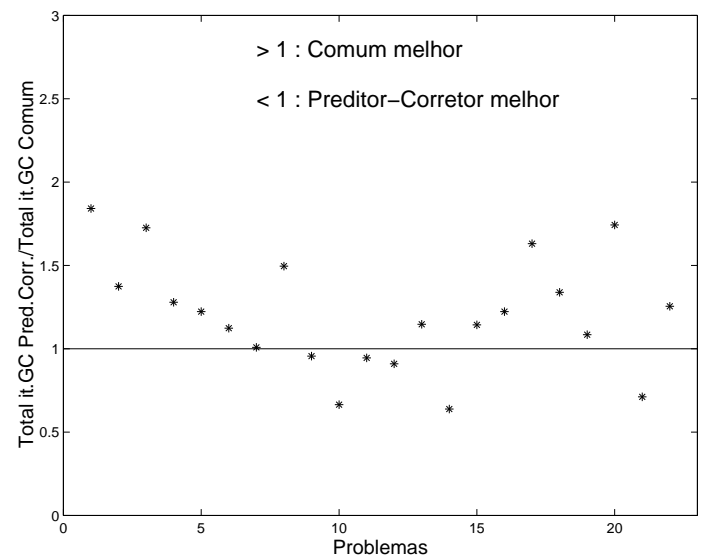

Figura 3: razão entre total de iterações no GC (ponto inicial I)

\begin{abstract}
In this paper we compare two specialized versions of the primal-dual interior point method for multiflow problems: one version called Usual and a second one known as Predictor-Corrector. We studied the cpu time, the number of iterations of the primal-dual method, as well as the total number of iterations of the preconditioned conjugate gradient in the presence of distinct starting points and different initializations in the preconditioned conjugate gradient.
\end{abstract}

\title{
Referências
}

[1] M. Kojima, N. Megiddo e S. Mizuno, A primal-pual infeasible-interior-point algorithm for linear programming, Mathematical Programming 61 (1993), 263280.

[2] M. Kojima, S. Mizuno e A. Yoshise, A primal-dual interior- point method for linear programming, em "Progress in Mathematical Programming" (N. Megiddo, ed.), pp. 29-48, Springer-Verlag, New York, 1989.

[3] I.J. Lustig, R.E. Marsten e D.F. Shanno, On implementing Mehrotra's predictor-corrector interior point method for linear programming, SIAM Journal on Optimization 2 (1992), 435-449.

[4] K.A. McShane, C.L. Monma e D.F. Shanno, An implementation of a primaldual interior point method for linear programming, ORSA Journal on Computing 1 (1989), 70-83.

[5] S. Mehrotra, On the implementation of a primal-dual interior point method, SIAM Journal on Optimization 2 (1992), 575-601.

[6] V. Podestá-Gomes, "Método Primal-Dual de Pontos Interiores Aplicado ao Problema de Multifluxo", Tese de Doutorado, UNICAMP, 1999. 